\title{
Formation of granular jets observed by high-speed X-ray radiography
} \author{
PETER J. ENG ${ }^{1,3}$ AND HEINRICH M. JAEGER ${ }^{1}$ \\ ${ }^{1}$ Department of Physics, James Franck Institute, The University of Chicago, Chicago, Illinois 60637, USA \\ ${ }^{2}$ Departmento de Fisica, Universidad de Chile, Casilla 487-3, Santiago, Chile \\ ${ }^{3}$ Consortium for Advanced Radiation Sources, The University of Chicago, Chicago, Illinois 60637, USA \\ *e-mail: jroyer@uchicago.edu
}

JOHN R. ROYER ${ }^{1 *}$, ERIC I. CORWIN ${ }^{1}$, ANDREW FLIOR ${ }^{1}$, MARIA-LUISA CORDERO², MARK L. RIVERS ${ }^{3}$,

W hen a heavy sphere is dropped onto a bed of loose, fine sand, a remarkable phenomenon occurs: a large, focused jet of sand shoots upwards ${ }^{1-4}$. Although similar looking jets are observed on impact in fluid systems $s^{5-7}$, they are held together by surface tension. Surprisingly, the granular jet exists in the absence of both surface tension and cohesion, thus fluid jet models are of limited use. Previous work $^{1,2}$ proposed that the jet is created solely by the gravity-driven collapse of a void left by the sphere's descent through the pack. Here we present experimental evidence that granular jets are instead driven by a more complex process involving the interaction between the sand and interstitial air. Using high-speed X-ray radiography, and high-speed digital video, we observe the formation of the jet both inside and above the bed. We find that what previously was thought of as a single jet in fact consists of two components: a wispy, thin jet that varies little with pressure followed by a thick air-pressure-driven jet. This is further evidence that qualitatively new phenomena in granular systems can emerge as a function of air pressure ${ }^{8-11}$. Our results highlight the importance of the dynamic coupling between gas and granule motion.

As discovered in ref. 1 and further studied in refs 2,3, granular jets can be observed following the impact of a heavy sphere into a sufficiently loose and fine-grained bed (typical grain diameters $100 \mu \mathrm{m}$ or below). Under these conditions, the bed is only marginally mechanically stable, and the impacting object can sink in easily. In fact, the jet is also observed if a heavy sphere is released from rest directly above the bed ${ }^{3}$. The main features of granular jets under atmospheric conditions are depicted in Fig. 1a-c. After an initial crown-shaped splash of grains from the bed's surface, the jet emerges from within the void created by the impacting sphere. It rises high into the air, with a velocity gradient along the height of the jet, creating a straining flow from top to bottom, ending with the break-up of the jet into discrete clumps of sand. Earlier reports of granular jets focused on the dependence of the maximum jet height on parameters such as the release height of the sphere. Using observations above bed level and quasi-two-dimensional setups with the bed material contained between two parallel glass walls, as well as simulations, these studies concluded that granular jets were produced when the void left by the impacting sphere collapsed under gravity ${ }^{1,2}$. In this scheme, the collapsing void walls behave in a similar manner to colliding liquid surfaces ${ }^{6}$. The role of gas is limited to providing a slight underpressure behind the impacting sphere, which can pull the upper edge of the splash 'corona' into the void (Fig. 1b), and to introducing drag on the particles.

Evacuation of the system (Fig. 1d-f) indeed reduces the particle drag and increases the size of the corona (Fig. 1e). However, we find that these effects pale in comparison with the marked reduction in the size of the jet itself (Fig. 1f). Furthermore, as the pressure is varied, a pronounced two-stage structure emerges (Fig. 2a-c), consisting of an initial thin jet, followed by an abrupt shoulder and a markedly thicker jet. Below about $5.3 \mathrm{kPa}$, only the thin jet remains visible, whereas above $67 \mathrm{kPa}$ the thin jet is subsumed into the thick jet.

By measuring the pressure dependence of the velocity of the two jets we can gain some insight into their respective origins. As seen from Fig. 2d, the thick jet's velocity decreases strongly with pressure, vanishing around $5.3 \mathrm{kPa}$. By contrast, the small jet's velocity varies by less than a factor of two down to the lowest pressures and approaches a finite value. We conclude that the thick jet requires air to form, whereas the thin jet will form even in the absence of air.

To observe the initial stages of jet formation inside the threedimensional granular bed, we used X-ray radiography using the high-intensity beams available at the Advanced Photon Source. This technique, used here for the first time at frame rates up to 5,000 frames per second, allowed us to image a twodimensional projection of the density inside the sand bed in real time (see Supplementary Information videos). These videos clearly demonstrate the formation and ejection of the granular jet. Figure 3 shows image sequences assembled from experimental runs for a $12 \mathrm{~mm}$ sphere in a $30 \mathrm{~mm}$ diameter cylindrical container at atmospheric pressure, where the effects of the interstitial gas are most pronounced. The sphere impacts the loosely packed bed and creates a cylindrical void (Fig. $3 \mathrm{a}$ ). The walls then collapse 
a

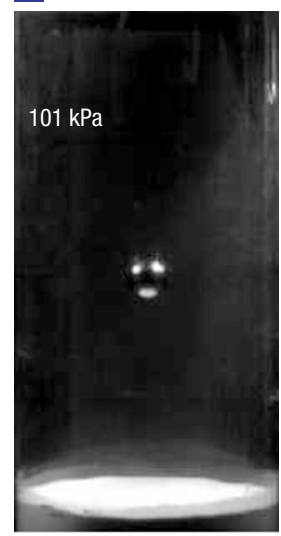

b

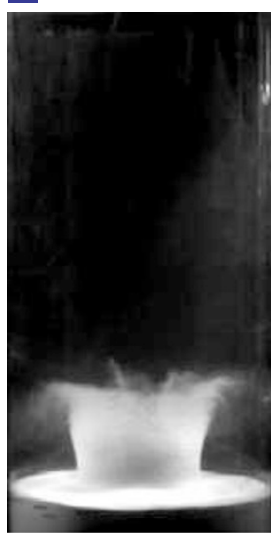

c

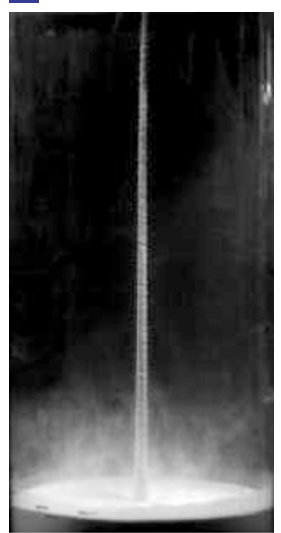

d

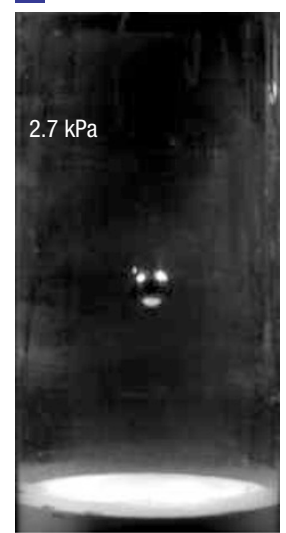

e

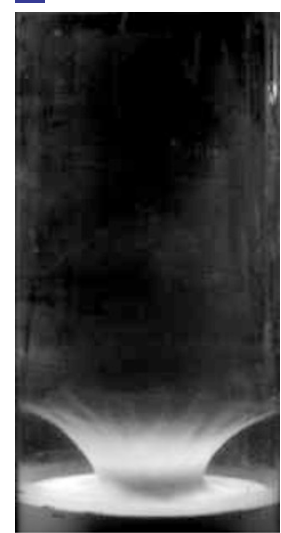

f

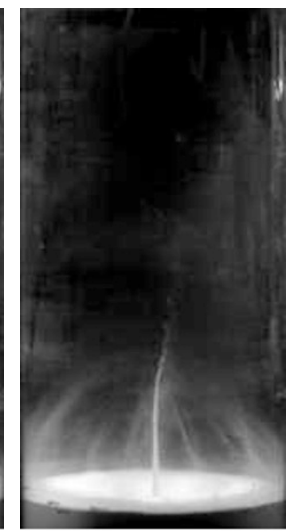

Figure 1 Comparison between jets at atmospheric pressure and vacuum. a-c, Images from a high-speed video of the jet at atmospheric pressure and $\mathbf{d}-\mathbf{f}$, at reduced pressure. Images $\mathbf{b}$ and $\mathbf{e}$, show the initial splashes $t=0.06 \mathrm{~s}$ after impact. $\mathbf{c}$, The jet at its maximum height at atmospheric pressure $t=0.31 \mathrm{~s}$ after impact and $\mathbf{f}$, at its maximum height in vacuum $t=0.18 \mathrm{~s}$ after impact. Air drag on the ball was negligible, so the impact velocity $\left(4.4 \mathrm{~m} \mathrm{~s}^{-1}\right)$ was independent of pressure. However, air drag had a substantial effect on the motion of the sand, which can be seen from the wider corona at reduced air pressure (e). In spite of this reduced air drag, the jet in the evacuated container is considerably thinner and smaller than the jet observed at atmospheric pressure.

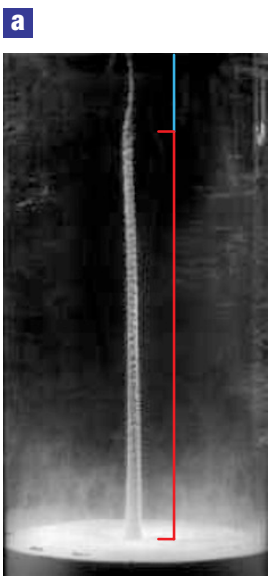

b

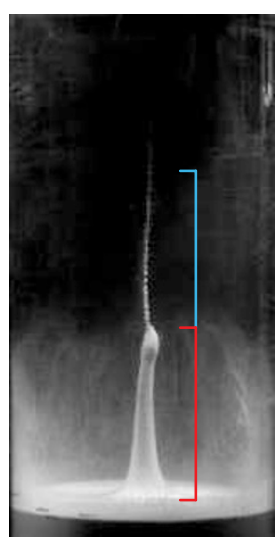

G

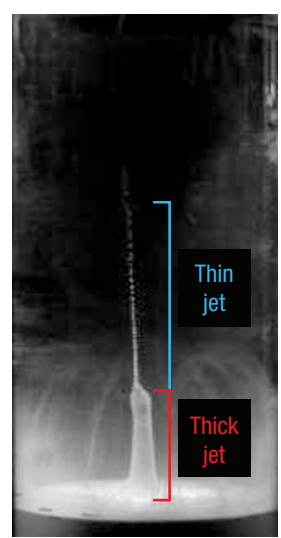

d

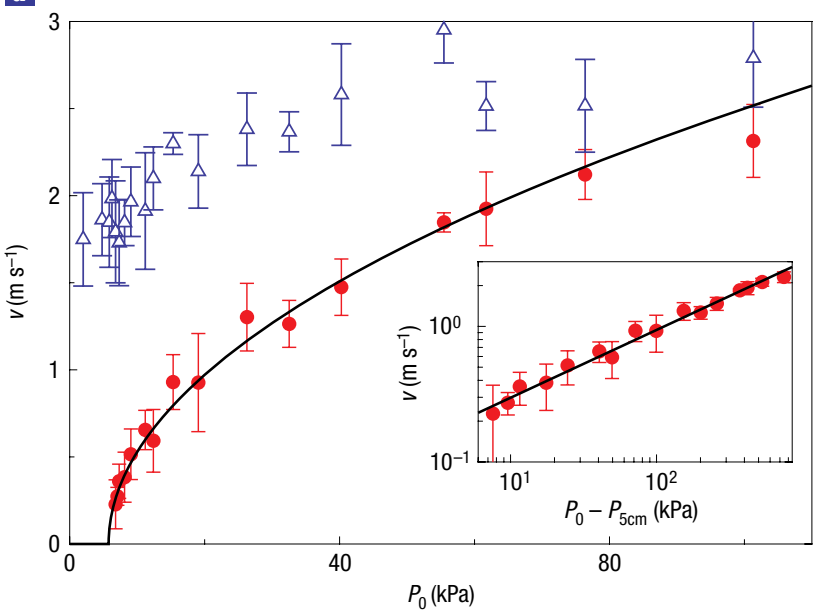

Figure 2 Pressure dependence of the thick and thin jets. The jet at its maximum height at a, $6.67 \mathrm{kPa}, \mathbf{b}, 2.67 \mathrm{kPa}$ and $\mathbf{c}, 1.33 \mathrm{kPa}$. The thick, air-dependent jet and thin air-independent jet are marked by red and blue sidebars. $\mathbf{d}$, Plot of the tip velocity $v$ of the thick jet (red circles) and thin jet (blue triangles) as a function of the gas pressure $P_{0}$. The tip velocity was measured $5 \mathrm{~cm}$ above the top surface of the sand so that the corona from the initial splash did not block the view of the jet. The tip velocity of the thick jet clearly goes to zero at a finite pressure, whereas the tip velocity of the thin jet only weakly depends on pressure and approaches a constant value as $P_{0} \rightarrow 0$. The tip velocity of the thick jet was fit to $v\left(P_{0}\right)=a\left(P_{0}-P_{5 \mathrm{~cm}}\right)^{1 / 2}$, shown by the solid line. The offset pressure $P_{5 \mathrm{~cm}}=5.7 \mathrm{kPa}$ corresponds to the pressure below which the thick jet did not reach $5 \mathrm{~cm}$ above the top surface. The inset is a log-log plot of the tip velocity of the thick jet as a function of $P_{0}-P_{5 \mathrm{~cm}}$. Error bars represent statistical errors from averaging multiple experiments.

radially into the void and collide along the central vertical axis. As suggested in ref. 2, the speed of the closing-up initially increases with depth below the surface. The collapsing walls therefore meet first at some point $z_{\mathrm{c}}$ below the surface (arrow in Fig. 3b) and produce a small upward jet (upper arrow in Fig. 3c). The thick jet, however, is not produced by the initial radial collapse, but instead by a subsequent process driven by the air trapped beneath the pinch-off (Fig. 3c-e).

The formation of the thick jet involves several qualitatively new features not contained in the simple inertial collapse model. First, inertial collapse predicts ${ }^{2}$ a single pinch-off at $z_{\mathrm{c}}$. Instead, our X-rays show a second pinch-off at the bottom of the cavity, resulting in an additional thin jet (lower arrow in Fig. 3c). Second, the thick jet only emerges after significant compression of the air pocket trapped below the uppermost pinch-off. Compression of the air pocket is driven by material collapsing into the void from the bottom, where the hydrostatic pressure is largest. As a result of the compression, the height of the cavity shrinks markedly, the cavity bulges outwards radially, and any small downward-facing jet is diminished (Fig. 3d). Simultaneously, the compressed gas pushes bed material up, which then forms the thick jet. The gas can even be seen breaking through the upper pinch-off, intruding into the thick 
jet and partially hollowing it out (Fig. 3e). The intrusion of air into the thick jet explains the peculiar shoulder seen in the thick jets in Fig. $2 \mathrm{~b}$ and $\mathrm{c}$, signalling the top of the thick jet.

Although air bubbles formed underneath a jet in liquid can decrease its energy ${ }^{6}$, in granular matter we find that the trapped air pocket is in fact the key source of energy for the thick jet. Guided by the compression and subsequent upward expansion of the cavity as visualized by the $\mathrm{X}$-rays videos, we treat the trapped gas pocket as a pressure spring. From this we can find the scaling of the thick jet's velocity, $v$, with ambient gas pressure, $P_{0}$. The energy from an initial, small compression of the air pocket, $\Delta V$, varies as $P_{0} \Delta V$. Assuming that this compression drives the initial ejection of the thick jet, we obtain $P_{0} \Delta V \sim E_{\text {tip }} \sim v^{2}$, where $E_{\text {tip }}$ is the initial kinetic energy of the jet tip, so that $v \propto \sqrt{P_{0}}$, in excellent agreement with the data in Fig. 2.

An overpressure capable of propelling the granular jet upwards can form in a cavity surrounded by a porous granular medium as soon as the cavity volume shrinks significantly faster than the gas can escape. To check this, we estimate both the required amount of overpressure and its build-up time. The shortest, and therefore easiest, path between the air pocket and the ambient air is through the thin plug of sand formed by the uppermost pinch-off. As a first approximation we only consider air flow through this plug and treat the cavity as a cylindrical piston with a constant radius and with the bottom moving up with constant velocity $v_{\mathrm{b}}$. From Darcy's law ${ }^{12,13}$, the flow velocity $u$ through a porous plug of thickness $s$ driven by a pressure difference $\Delta P$ is $u \approx(k / \mu s) \Delta P$. Here $\mu$ is the viscosity of the gas and $k$ is the permeability of the granular material, which we express in terms of the packing fraction $\phi$ and grain diameter $d$ using the Carman-Kozeny relation ${ }^{13}$ $k=d^{2}(1-\phi)^{3} / 180 \phi^{2}$.

If $u$ is comparable to $v_{\mathrm{b}}$ then air can easily escape through the top and no overpressure will build. However, if $u \ll v_{\mathrm{b}}$, then the cavity will rapidly pressurize. In our experiment $\mu=1.8 \times 10^{-5} \mathrm{~Pa}, d=50 \mu \mathrm{m}$ and $\phi \sim 0.55$. From the X-ray videos we measure $s \sim 10 \mathrm{~mm}$ and $v_{\mathrm{b}} \sim 1 \mathrm{~m} \mathrm{~s}^{-1}$. For these conditions we find that $u \ll v_{\mathrm{b}}$ as long as $\Delta P \ll 42 \mathrm{kPa}$. On the other hand, the pressure difference required to support the weight of the plug is only $\Delta P_{\mathrm{w}}=\rho_{\mathrm{s}} \phi g s \cong 0.13 \mathrm{kPa}$, where $\rho_{\mathrm{s}}=2.5 \mathrm{~g} \mathrm{~cm}^{-3}$ is the density of the sand and $g$ the acceleration due to gravity. For short times before the plug moves appreciably, the pressure difference across the plug rapidly increases according to $\Delta P \approx P_{0} t / t^{*}$. Here $t=0$ is the time when the top starts to pinch-off and, in our simple model, $t^{*}=h_{0} / v_{\mathrm{b}}$ is the timescale for complete collapse of a cavity of initial height $h_{0}$. From our X-ray data $h_{0} \sim 30 \mathrm{~mm}$ so that the pressure difference $\Delta P_{\mathrm{w}}$ required to support the weight of the plug builds up in as little as $7 \times 10^{-5} \mathrm{~s}$.

In the above estimates, we have ignored diffusion of air into the rest of the bed. However, we can show that such diffusion will not relax $\Delta P$ significantly. One can derive ${ }^{13}$ a diffusion equation from Darcy's law and the continuity equation with a diffusion constant $D=k P_{0} / \mu(1-\phi)$, which is about $0.05 \mathrm{~m}^{2} \mathrm{~s}^{-1}$ for our conditions. The characteristic time for air to diffuse to the walls of the container (a distance $l \sim 10 \mathrm{~mm}$ ) is $\tau_{\mathrm{d}}=l^{2} / D \sim 0.002 \mathrm{~s}$. This time is much longer than the time for the pressure to increase to $\Delta P_{\mathrm{w}}$. In a time $\tau_{\mathrm{d}}$ the pressure difference would already have reached about $4 \mathrm{kPa}$ or 30 times $\Delta P_{\mathrm{w}}$. Thus, an overpressure capable of driving the plug upwards will develop long before outflow of air through the plug or the bed can relax the pressure gradient.

However, if the bed permeability becomes too large, either because $d$ is increased or because $\phi$ decreases locally owing to the presence of nearby walls ${ }^{14}$, trapped gas can much more easily escape. In this case, increased outflow through the sand bed prevents the build-up of a pressure difference capable of driving the plug upwards. We believe that this is why neither the simulations

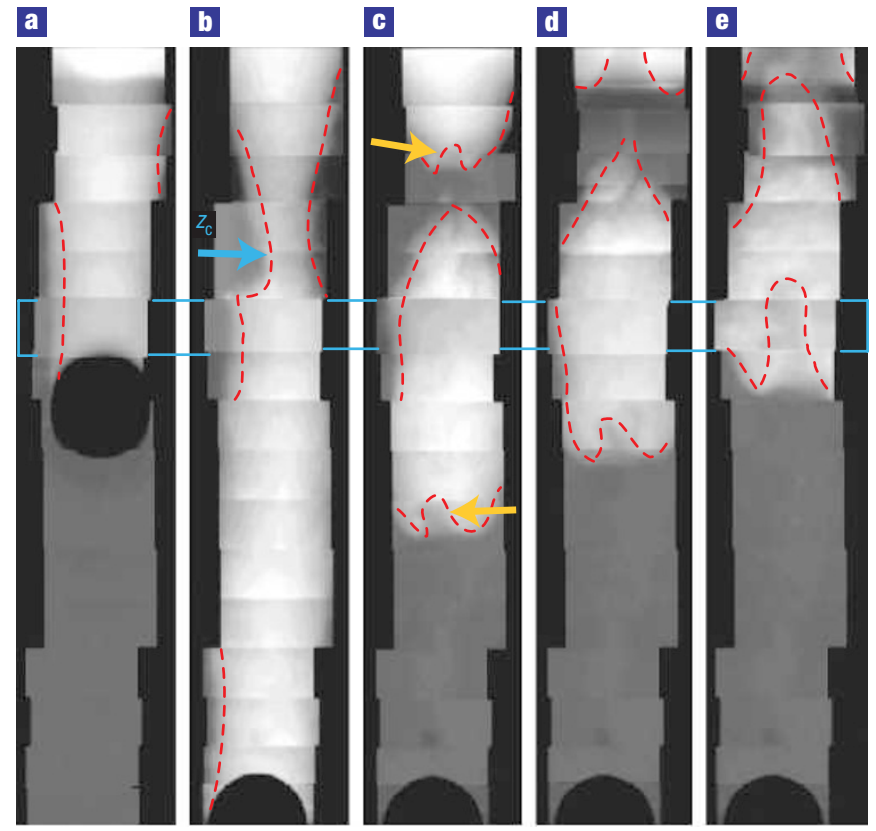

Figure 3 Reconstructed X-ray radiographs of the interior of the pack. Images taken from the reconstructed video at a, $t=0.01 \mathrm{~s}, \mathbf{b}, t=0.07 \mathrm{~s}, \mathbf{c}, t=0.10 \mathrm{~s}$, d, $t=0.11 \mathrm{~s}$ and $\mathbf{e}, t=0.14 \mathrm{~s}$ after the sphere impacted the top surface. The cavity left by the ball, highlighted by the red dashed line, appears as a light region against the grey background of boron carbide. The video taken $55 \mathrm{~mm}$ above the bottom did not match the other videos, owing to insufficient aeration, so for clarity images from the video $5 \mathrm{~mm}$ below were used to match the profile of the cavity (bracketed by a blue rectangle). The base of the main jet, as well as the smaller jets protruding into the cavity, can be seen and also are highlighted by the dashed line. The vertical position of the upper pinch-off, $z_{\mathrm{c}}$, is marked by a light-blue arrow in $\mathbf{b}$. The initial thin jet formed by the upper pinch-off and hidden thin jet formed by the lower pinch-off are marked by orange arrows in $\mathbf{c}$.

nor the quasi-two-dimensional experiments in ref. 2 show evidence of the thick jet seen in three-dimensional experiments, but instead only the thin jet.

From the X-ray videos and this simple model, we posit a complete sequence of the formation of granular jets in three dimensions. The sphere impacts the bed and creates a cylindrical void. This void collapses owing to hydrostatic pressure and forms several pinch-offs. The radially symmetric influx of sand into the cavity region demands that collision momentum must escape axially ${ }^{2}$. The closing of the cavity at the uppermost pinch-off point initially gives rise to the thin jet. This pinch-off subsequently traps an air pocket below the surface. In the presence of sufficiently high ambient pressure, the trapped air pocket can develop a large overpressure. This propels a thick jet by driving upwards the sand flowing radially into the upper pinch-off.

It has been suggested that these small-scale impact experiments could serve as a model for crater formation and material ejection due to large-scale astrophysical impacts ${ }^{1,2,4}$. Our findings show that gas is important in the formation of a granular jet, adding a key parameter that may only be present in certain types of astrophysical impact. Perhaps granular impact experiments done under vacuum can serve as a more suitable model.

In the splash region, non-cohesive granular material is easily dispersed into a fine spray. However, the thick jet remains remarkably collimated as it drives upwards. Although we have uncovered the forcing mechanism driving the phenomenon, the 
processes that maintain the well-defined, collimated shape of the jet as well as the eventual break-up into compact droplets remain unclear. High-speed X-ray imaging shows that the interaction between the grains and interstitial gas inside the bed can produce pressure gradients that stabilize the sharply delineated, shock-like boundaries between the sand and air pocket. We speculate that the straining flow experienced by the upward-moving jet creates an underpressure inside the jet similarly responsible for maintaining the jet's sharp boundaries as it rises above the bed. In this way, airpressure gradients can exert force on and stabilize a boundary. This effect may play a role in granular systems similar to surface tension in fluids.

\section{METHODS}

For optical measurements of the jet above the bed surface, a 22-mm steel ball was dropped from $1.0 \mathrm{~m}$ into a 20 -cm-deep bed of spherical glass beads ( $d=50 \mu \mathrm{m}$, angle of repose $\sim 26^{\circ}$ ) in a cylindrical vessel with $15-\mathrm{cm}$ inner diameter. The resulting jet imaged at up to 5,000 frames per second using a high-speed video camera (Phantom v7.1). The system could be sealed and evacuated to explore the role of air pressure. Before each drop, the bed was prepared reproducibly in a low-density state (packing fraction around $\phi=0.55$ ) by aerating it from below with dry nitrogen. After turning off the nitrogen flow and evacuating the system to the desired pressure, the heavy sphere was dropped. We verified that the pumping did not disturb the loose packing by slowly cycling from atmospheric conditions to our minimum pressure $(2 \mathrm{kPa})$ and back, and then performing a drop without observing any change in the jet dynamics.

High-speed X-ray radiographs were carried out using a high-intensity 'pink' X-ray beam with an energy width of about $5 \mathrm{keV}$ centred at $22 \mathrm{keV}$ at the University of Chicago's GeoSoilEnviroCARS bending magnet beamline (13 BMD) at the Advanced Photon Source. To image the interior, there must be appreciable X-ray transmission through the sand bed. This forced us to use a thinner container and a granular material with a lower atomic number. For the $\mathrm{X}$-ray images presented here, a $12-\mathrm{mm}$ steel ball was dropped from $34 \mathrm{~cm}$ into a 75 -mm-deep bed of $d=50 \mu \mathrm{m}$ boron carbide $\left(\mathrm{B}_{4} \mathrm{C}\right)$ particles, contained in a cylinder with inner diameter $30 \mathrm{~mm}$. The $\mathrm{B}_{4} \mathrm{C}$ particles where rough and non-spherical and had a higher angle of repose $\left(\sim 34^{\circ}\right)$. Despite the smaller container and different material, optical videos of the jet above the surface showed no qualitative differences between the jets in the two setups (see Supplementary Information videos). The bed was fluffed by air entering through a diffuser built into the bottom of the cylinder. The local X-ray transmission through the bed was imaged off a phosphor screen at up to 5,000 frames per second. The beam size limited the field of view to $15 \mathrm{~mm} \times 9 \mathrm{~mm}$ sections of the container. Radiographs of the full column were reconstructed by assembling images from several, independent drops, each taken at a different vertical position and synchronized using the passage of the ball as a guide. Although the impact position varied slightly from drop to drop, the excellent image overlap in the vertical direction attests to the high degree of reproducibility between subsequent drops.

The X-ray optics used to spread the beam created an energy gradient across the height of the beam, resulting in raw images that were brighter at the top than at the bottom. Each video was divided by a background image taken before the ball passed through to remove this variation in intensity. However, as the $\mathrm{X}$-ray transmission profile depends on the amount of material the X-rays pass through, this background division overcompensates in the regions with less material. This creates a slight vertical intensity gradient in images of the cavity.

Received 29 July 2005; accepted 31 October 2005; published 1 December 2005.

References

1. Thoroddsen, S. T. \& Shen, A. Q. Granular jets. Phys. Fluids 13, 4-6 (2001).

2. Lohse, D. et al. Impact on soft sand: Void collapse and jet formation. Phys. Rev. Lett. 93 198003 (2004).

3. Lohse, D., Rauhe, R., Bergmann, R. \& van der Meer, D. Creating a dry variety of quicksand. Nature 432, 689-690 (2004).

4. Walsh, A. M., Holloway, K. E., Habdas, P. \& de Bruyn, J. R. Morphology and scaling of impact craters in granular media. Phys. Rev. Lett. 91, 104301 (2003).

5. Worthington, A. M. A Study of Splashes (Longman and Green, London, 1908).

6. Zeff, B. W., Kleber, B., Fineberg, J. \& Lathrop, D. P. Singularity dynamics in curvature collapse and jet eruption on a fluid surface. Nature 403, 401-404 (2000).

7. Fedorchenko, A. I. \& Wang, A. B. On some common features of drop impact on liquid surfaces. Phys. Fluids 16, 1349-1365 (2004).

8. Pak, H. K., Vandoorn, E. \& Behringer, R. P. Effects of ambient gases on granular-materials under vertical vibration. Phys. Rev. Lett. 74, 4643-4646 (1995).

9. Möbius, M. E., Cheng, X., Karczmar, G. K., Nagel, S. R. \& Jaeger, H. M. Intruders in the dust Air-driven granular size seperation. Phys. Rev. Lett. 93, 198001 (2004).

10. Yan, X., Shi, Q., Hou, M., Lu, K. \& Chan, C. K. Effects of air on the segregation of particles in a shaken granular bed. Phys. Rev. Lett. 91, 014302 (2003).

11. Burtally, N., King, P. J. \& Swift, M. R. Spontaneous air-driven separation in vertically vibrated fine granular mixtures. Science 295, 1877-1879 (2002).

12. Darcy, H. P. G. Les Fontaines Publiques de la Ville de Dijon (Victor Dalmont, Paris, 1856).

13. Carman, P. C. Flow of Gases Through Porous Media (Butterworths Scientific, London, 1956).

14. Dullien, F. A. L. in Handbook of Powder Science and Technology (eds Fayed, M. E. \& Otten, L.) (Van Nostrand Reinhold, New York, 1984).

\section{Acknowledgements}

We thank F. Blanchette, E. Clement, B. Conyers, K. Foster, M. Möbius and S. Nagel for insightful discussions. This work was supported by NSF through its MRSEC program under DMR-0213745 and through its Inter-American Materials Collaboration under DMR-0303072. GSECARS is supported by National Science Foundation-Earth Sciences (EAR-0217473), Department of Energy Geosciences (DE-FG02-94ER14466), the State of Illinois and the W. M. Keck Foundation. Use of the Advanced Photon Source was supported by the US Department of Energy, Basic Energy Sciences, Office of Energy Research, under contract number W-31-109-Eng-38.

Correspondence and requests for materials should be addressed to J.R.R

Supplementary Information accompanies this paper on www.nature.com/naturephysics.

Competing financial interests

The authors declare that they have no competing financial interests.

Reprints and permission information is available online at http://npg.nature.com/reprintsandpermissions/ 